Meta

Journal des traducteurs

Translators' Journal

\title{
Traduire l'Angleterre sous la Restauration : Gibbon et Shakespeare de Guizot
}

\section{Gabriel Louis Moyal}

Volume 50, numéro 3, août 2005

Le prisme de l'histoire

The History Lens

URI : https://id.erudit.org/iderudit/011603ar

DOI : https://doi.org/10.7202/011603ar

Aller au sommaire du numéro

Éditeur(s)

Les Presses de l'Université de Montréal

ISSN

0026-0452 (imprimé)

1492-1421 (numérique)

Découvrir la revue

Citer cet article

Moyal, G. L. (2005). Traduire l'Angleterre sous la Restauration : Gibbon et Shakespeare de Guizot. Meta, 50(3), 881-905. https://doi.org/10.7202/011603ar
Résumé de l'article

La carrière de François Guizot - historien, théoricien du libéralisme, premier ministre - a laissé dans l'ombre ses travaux de traducteur. Angliciste reconnu, Guizot a traduit l'Histoire de la décadence et de la chute de l'Empire romain de Gibbon ainsi que les oeuvres complètes de Shakespeare. Chacune de ces traductions lui servait aussi à transmettre son idéologie. La traduction de l'ouvrage de Gibbon instaurait des normes épistémologiques pour la traduction : la nécessité d'expliciter pour produire une traduction précise. L'introduction à la traduction de Shakespeare, en liant l'histoire du théâtre à l'évolution politique, proposait le modèle constitutionnel anglais comme solution politique viable pour la France de la Restauration.
Ce document est protégé par la loi sur le droit d'auteur. L'utilisation des services d'Érudit (y compris la reproduction) est assujettie à sa politique d'utilisation que vous pouvez consulter en ligne.

https://apropos.erudit.org/fr/usagers/politique-dutilisation/ 


\title{
Traduire l'Angleterre sous la Restauration: Gibbon et Shakespeare de Guizot
}

\author{
GABRIEL LOUIS MOYAL \\ McMaster University, Hamilton, Canada \\ moyalg@univmail.cis.mcmaster.ca
}

\begin{abstract}
RÉSUMÉ
La carrière de François Guizot - historien, théoricien du libéralisme, premier ministre a laissé dans l'ombre ses travaux de traducteur. Angliciste reconnu, Guizot a traduit l'Histoire de la décadence et de la chute de l'Empire romain de Gibbon ainsi que les œuvres complètes de Shakespeare. Chacune de ces traductions lui servait aussi à transmettre son idéologie. La traduction de l'ouvrage de Gibbon instaurait des normes épistémologiques pour la traduction: la nécessité d'expliciter pour produire une traduction précise. L'introduction à la traduction de Shakespeare, en liant l'histoire du théâtre à l'évolution politique, proposait le modèle constitutionnel anglais comme solution politique viable pour la France de la Restauration.
\end{abstract}

\section{ABSTRACT}

François Guizot's parallel careers as political theorist, prime minister of France and major historian have nearly eclipsed his contributions as a translator. An eminent anglicist, Guizot translated both Gibbon's Decline and Fall of the Roman Empire and Shakespeare's complete works into French. These translations served to convey Guizot's predominant ideological concerns. The Gibbon translation established epistemological norms for translation: the necessity of erudite elaboration to produce accurate translation. The Shakespeare translation's introduction bound comparative history of the theatre with political evolution to subtly promote the British constitutional model as a credible political alternative.

\section{MOTS-CLÉS/KEYWORDS}

François Guizot, Gibbon, Restauration, Shakespeare

\section{Introduction}

J'ai eu plus d'une fois l'occasion de déplorer l'entêtement qui nous fait négliger, plus encore par légèreté que par dédain, l'étude des littératures étrangères. Quelques imitations de Shakspeare [sic] ont eu du succès sur notre théâtre; mais a-t-on cherché à connaître le caractère particulier de ce poète, à approfondir le système dramatique de sa nation? a-t-on examiné à quel ensemble de principes et d'idées ce système était lié? a-t-on comparé ces principes avec ceux sur lesquels s'est formée notre poésie? s'est-on appliqué à voir ce qu'ils avaient d'incompatible? a-t-on tiré de là un tableau des avantages et des inconvénients des deux systèmes? Cette carrière ouverte à la sagacité des critiques, à l'analyse des littérateurs philosophes, semble avoir à peine été tentée; et cependant de telles recherches sont propres à jeter un grand jour, non seulement sur les divers genres de poésie dramatique, mais encore sur l'histoire et le caractère des nations: tout se tient dans la nature, et plus qu'aucune autre branche des belles-lettres, la poésie dramatique est liée de près aux opinions et aux mœurs ${ }^{1}$. 
François Guizot (1787-1874) entamait ainsi, en 1809, son long compte rendu sur la traduction récente par Benjamin Constant du Wallenstein de Schiller. Cette entrée en matière en dit long sur la façon dont Guizot concevait en général le rôle et la tâche du traducteur. Il situe d'emblée le débat sur la traduction au-delà des questions de style et d'expression. De même, le débat sur les règles et traditions théâtrales est vite mis de côté. Guizot va droit à la confrontation de cultures entières - voire de civilisations - qu'implique la traduction. Avancer comme il le fait ici que «tout se tient dans la nature» dans ce contexte de recoupement qu'occasionnent les traductions c'est laisser entendre qu'il préconise pour la traduction un programme bien plus vaste que celui que rhétoriciens et traducteurs entendaient alors qu'ils débattaient - et continuaient de débattre - les conflits entre forme et sens en traduction. Ce «tout se tient dans la nature» n'est pas simplement ici une proclamation d'appartenance, un mot d'ordre de la tradition philosophique et encyclopédique des Lumières. C'est une déclaration de principe: Guizot annonce ses attentes, sa façon de concevoir, par le biais de cette critique d'une traduction, les responsabilités, les devoirs des traducteurs.

Et si ces attentes peuvent paraître excessives, si, par exemple, il parle ici d' «imitations» et semble par là ignorer ou mépriser les traductions existantes de Shakespeare, il ne faudrait pas présumer de cela que Guizot ne parle que dans l'abstrait. Peu après avoir écrit ces lignes, Guizot allait acquérir une expérience pratique de la traduction - et le droit d'en parler. Entre 1810 et 1812, il allait traduire et très amplement annoter l'Histoire de la décadence et de la chute de l'Empire romain de Gibbon. Très vite, ses notes, questions et commentaires se trouveront traduits en anglais et intégrés dans les grandes éditions subséquentes de l'œuvre de Gibbon².

Mais il transparaît de ce passage que, pour Guizot, il ne saurait suffire pour traduire que de traduire les mots, ou même un texte entier. Comme la nature qui se lie, le savoir, qui veut en être le reflet fidèle, doit ambitionner de tout comprendre et de là chercher à tout faire comprendre. Dès lors, aucun détail n'est en fin de compte insignifiant, il n'y a plus de marge d'exclusion ou de pris pour acquis: tout se relie et les liens sont à démontrer in extenso ${ }^{3}$. Et, pour Guizot, la traduction a pour tâche particulière d'élaborer ces liens, d'exposer - dans et entre les deux cultures qu'elle relie - les points de rapport, les différences et les fausses autant que les vraies ressemblances.

Tout peut toujours s'expliciter chez Guizot, pourvu que nous prenions ou qu'il nous soit accordé au préalable le temps de bien comprendre. La question de l'intraduisible, de l'impossibilité de rendre tel effet de style ou, telle nuance de forme ou de sens ne se pose pratiquement pas. Aucun tribut n'est à rendre à de mystérieuses subtilités supposées de la langue de départ. Aucune concession ne sera faite aux états d'âme du traducteur qui se veut aussi fin poète que l'auteur qu'il traduit: tout peut se rendre en long et en large, il suffit d'expliquer sans rien omettre tout ce que l'on aura compris de l'original. Une traduction qui viserait moins ne vaudrait pas aux yeux de Guizot une humble «imitation». La responsabilité de rendre un sens entier l'emporte sans hésitation sur la fidélité au style et à ses caprices. Et en autant que le style peut affecter le sens les effets en sont d'autant mieux explicitables. La responsabilité didactique qu'il assume primera partout les exigences de l'esthétique, du goût ou des règles données pour classiques. En fin de compte, la pédagogie devra informer l'esthétique ${ }^{4}$.

Mais l'ordre de valeurs qui dicte les attentes de Guizot tient sans doute plus à sa perspective historique particulière qu'à la seule pratique de la traduction. La logique de l'histoire ne se laisse entendre qu'une fois comprise comme un ensemble, comme 
un long continuum, dont les vraies extrémités sont hors de notre portée. Nous ne pouvons en apercevoir que de très vagues tendances en en découpant des segments (mouvement vers la civilisation, vers une répartition plus équitable ou démocratique des pouvoirs, etc.). Mais ces tendances, vaguement aperçues, présument de vastes accumulations de faits et surtout de la capacité de les classer et de les interpréter, de les traduire en leçons. Et, dans la conjoncture politique dans laquelle Guizot écrit, toute interprétation s'identifie bon gré, mal gré à une idéologie. Guizot n’hésite pas à prendre son parti. Son interprétation de l'histoire informe l'idéologie qui à son tour informe la tâche didactique qu'il se donne.

Mais il faut aussi considérer qu'il parle ici de "poésie dramatique», car tout ce qui concerne le théâtre occupe chez Guizot une place à part. Tel qu'il le conçoit, le théâtre renvoie infailliblement à la vie publique des nations. Le théâtre est pour Guizot moins significatif en tant qu'objet d'étude littéraire qu'il ne l'est en tant qu'objet historique. En tant que rituel, en tant qu'occasion de mise en présence de collectivités, de communautés, le théâtre est pour lui un lieu privilégié. Rituel public autant que spectacle, le théâtre devient comme la métonymie de la société où il se donne et des relations entre ses classes. L'évolution de ce rituel ne peut que suivre et refléter cette histoire sociale et finalement politique. Enfin, pour le Guizot historien, le texte dramatique est d'abord significatif en autant qu'il tienne compte de ces relations, qu'il s'adresse aux conflits, aux aspirations qui se confrontent tacitement dans la salle. Traduire la poésie dramatique d'une nation, selon cette perspective, rend avant toute autre chose essentielle l'intégration, la compréhension de cette histoire sociale et politique.

C'est pourquoi, quand il entreprendra de traduire Shakespeare, Guizot se sentira moins concerné par les textes des pièces. Il laissera ce soin à sa femme, Pauline de Meulan, et à son collaborateur, l'angliciste Amédée Pichot - quitte à revoir et à modifier leurs travaux. Il se chargera avant tout de la rédaction des deux introductions, longues d'un volume chacune, qui précéderont les deux éditions, ainsi que des préfaces qu'il ajoutera à chacune des pièces.

En rédigeant sa première introduction, l'intention de Guizot est que son Shakespeare et son histoire du théâtre en Angleterre ne serviront pas uniquement à expliquer une autre conception du théâtre. Il cherche surtout à ce que sa traduction introduise l'idée d'une autre structure politique et d'une autre façon de concevoir les rapports entre les classes qui, selon lui, sont véhiculés par les pièces. C’est pourquoi, tout au long de sa biographie de Shakespeare et de son histoire du théâtre en Angleterre, Guizot marque en parallèle, et étape par étape, l'évolution relativement calme de l'Angleterre, la répartition progressive des pouvoirs politiques entre la monarchie et les différentes strates de la société anglaise. En présentant sa traduction de Shakespeare, Guizot s'acharnera à rendre vraisemblable, réalisable, cette autre forme d'évolution politique à un public français qui vient de traverser les séismes de la Révolution et de l'Empire et qui s'installe mal dans le système constitutionnel qui lui a été imposé. Guizot avait visiblement bien compris le potentiel politique de la traduction, et la place privilégiée d'analyste des rapports entre pouvoir et culture qu'occupe tout traducteur qui a à transiger entre cultures et pouvoirs ${ }^{5}$. C'est par un choix délibéré, par une conviction idéologique exacerbée par une conjoncture politique qui tend à l'exclure du pouvoir, que Guizot traduit et présente Shakespeare de la façon dont il le fait ${ }^{6}$. 
Guizot ne pouvait raisonnablement s'attendre à ce que le théâtre en France, tel qu'il se trouvait structuré à l'époque, puisse d'emblée transmettre l'interprétation, le message intriqué qu'il voulait lui faire porter en traduisant Shakespeare. Shakespeare n'était finalement jouable que sous la forme d'imitations ou d'adaptations. Mais il reste que si la traduction de Shakespeare que Guizot a fini par produire se prête moins à être jouée qu'à être lue c'est aussi parce qu'elle est structurée d'abord pour répondre et réagir à des circonstances historiques, aux faits d'une histoire dont elle devait contribuer à changer le cours.

\section{Section I : Photographie et image de la traduction}

«Maintenant que l'intelligence et l'amour des littératures étrangères se sont répandus en France, maintenant que Shakspeare est familier à tous les esprits cultivés; un traducteur peut oser davantage et serrer le texte au plus près. Rien n'empêche aujourd'hui les traductions d'être aussi exactes qu'elles pourront jamais l'être; la tentation et le péril sont plutôt d'exagérer que d'atténuer les textes en les interprétant, et de faire des traductions pareilles à la photographie qui grossit les traits saillants des visages qu'elle reproduit ${ }^{7}$.»

Ce passage est tiré d'une brochure publicitaire publiée en 1851 par Didier et Cie. Elle annonce la parution d'une nouvelle édition - revue, corrigée et augmentée - de la traduction des œuvres de Shakespeare par François Guizot. La première édition de cette traduction avait été publiée presque trente ans plus tôt et avait eu un certain retentissement dans le contexte des confrontations qui s'entamaient alors entre un romantisme français encore naissant et un classicisme toujours solidement établi et méfiant. Stendhal, dans les lettres de Paris qu'il rédigeait pour la London Literary Gazette, signala à l'époque ce succès comme une nouvelle victoire romantique, victoire selon lui autant littéraire que politique ${ }^{8}$.

En ce qui concerne la politique, la brochure de 1851 est pour sa part plus nuancée. Elle rappelle certes la contribution de la traduction de 1821 au succès des «idées libérales en littérature» (Brochure, p. 3), mais elle le fait en fin de texte, comme par après coup, ayant insisté surtout dans ses deux premières pages sur la qualité et la fidélité de la traduction en soi ${ }^{9}$.

Cela dit, la comparaison de la traduction à une image photographique sur quoi la brochure aboutit s'insère de façon presque incontournable dans le discours savant de cette partie du dix-neuvième siècle. Depuis son invention, une vingtaine d'années plus tôt, la photographie hante toute discussion de la représentation. Mais, comme toute innovation, elle demeure pour un temps suspecte. Et la suspicion s'exprime parfois en termes contradictoires: la photographie est à la fois trompeuse (elle « grossit les traits saillants ») ou elle est trop banale parce que trop littéralement fidèle à la réalité qu'elle représente. Ce que l'on retrouve aussi dans un autre passage, mieux connu, de l'article «TRADUCTION» du Grand Dictionnaire Universel de Pierre Larousse:

«Aujourd'hui même, la question, bien que fort avancée dans le sens de la reproduction exacte des modèles, n'est pas entièrement résolue. Sans doute, quand il est possible de photographier littéralement l'original et en même temps d'y ajouter la couleur et la vie; quand il est possible d'unir la peinture au décalque pour accroître la vérité, personne n'hésite et ne demande mieux. Mais le plus souvent il faut se résigner à n'avoir que la photographie dans sa froide exactitude, ou la recherche de la couleur, du sentiment général aux dépens de la littéralité ${ }^{10}$.» 
Ainsi, en ce qui concerne l'auteur de l'article, une traduction équivalente à un décalque colorié vaudrait mieux que la «froide exactitude» de la photographie. Pris dans un sens général, ce genre de discours - les avancées technologiques sont, en ce qui concerne le travail d'interprétation, une fausse piste - trouverait sans doute des échos dans le débat sans fin sur la traduction par machine. Mais il serait plus difficile de se représenter précisément - hormis ce rejet sommaire d'une littéralité trop étroitement définie - exactement ce qu'entend l'auteur de l'article du Grand Dictionnaire.

Par contre, en ce qui concerne la brochure publicitaire, il nous semble utile de ne pas négliger cette comparaison de la traduction à la photographie, même si elle peut paraître marginale, voire accidentelle. Car dès que l'on commence à en déployer les implications, à tenter d'en reconstruire l'intention critique, on s'aperçoit qu'elle correspond à une sorte de résistance à une mode, à un discours positiviste qui commence à dominer l'investigation scientifique et qui trouve ses emblèmes dans des objets représentatifs du progrès technologique tels la photographie. La brochure nous semble aussi résumer une position par rapport à la traduction - ses présupposés, ses horizons - que l'on retrouve à la fois inscrite entre les lignes ou élaborée dans d'autres travaux de traduction de Guizot. La brochure n'est pas signée, mais on ne saurait penser que Guizot aurait pu accepter de la laisser publier s'il ne l'avait pas lui-même rédigée ou s'il n'avait tout au moins participé à sa composition ${ }^{11}$. Or il se trouve que lorsque l'on revient aux textes de Guizot lui-même sur la traduction, lorsque l'on consulte ses préfaces, ses introductions aux traductions (celle de Shakespeare et de Gibbon, entre autres), on s'aperçoit que la précision, qu'une grande fidélité à l'original, sont tout au contraire des qualités auxquelles il accorde la plus grande importance. Déjà, lorsque l'on resitue dans un contexte plus étendu les remarques de la brochure sur la photographie, il s'avère que ce ne sont ni la précision ni la fidélité en ellesmêmes qui sont visées. Ainsi, là où la brochure fait l'inventaire des modifications déjà opérées à partir de la traduction de Letourneur, et cela dès la traduction de Guizot de 1821, on voit que le souci de Guizot avait toujours été de restituer à la fois l'intégralité de l'œuvre autant dans la quantité des textes que dans les qualités du style:

[...] une tragédie entière, et deux poëmes dont Letourneur n'avait rien donné, étaient ajoutés; tous les passages que Letourneur avait supprimés dans le corps des pièces étaient rétablis, et cela seul rendait à Shakspeare au moins deux volumes de ses œuvres qu'il avait perdu en chemin; mais surtout la traduction avait été entièrement revue et corrigée d'après le texte, et si le nom de Letourneur était maintenu sur le titre, son système d'interprétation était détruit presque à chaque ligne. Ses infidélités déclamatoires ou timides avaient disparu, pour faire place à une exactitude, à une simplicité, à une hardiesse qui changeaient du tout au tout la physionomie du style et qui donnaient dès lors à la France « un vrai Shakspeare, sans déguisement et sans retranchement » (Brochure: 2).

Ainsi, dans la brochure, le nouveau et «vrai Shakspeare», offert maintenant (et depuis 1821) à la France, présenterait le genre même d'exactitude, de précision objective, qu'une première lecture de la critique de la photographie semblait invalider.

L'apparente contradiction nous amène donc à chercher une redéfinition de la distorsion que semblait d'abord viser la critique de la photographie: quels «traits saillants » s'agissait-il de ne pas grossir dans cette traduction de Shakespeare? Quelles exagérations fallait-il éviter tout en cherchant l'exactitude dans la traduction? En concluant, la brochure nommait la précision photographique une «infidélité d'une nouvelle sorte» et donnait la traduction de Guizot comme ne donnant pas dans le 
piège de «faire un Shakspeare français plus anglais et plus Shakspearien que le Shakspeare anglais lui-même»(Brochure: 3). Pourquoi enfin ce rejet de l'image photographique comme modèle de précision?

\section{Section II : Restauration et traduction}

Sans doute le calque colorié dont parle le Grand Dictionnaire serait-il implicitement plus proche de la traduction idéale visée par l'auteur de la brochure. Mais pour entendre cette critique de la traduction "photographique» en soi, il vaut la peine de retourner au contexte politique de l'édition de 1821 de la traduction de Shakespeare par Guizot - contexte que la brochure nous incite plus particulièrement à considérer par cette conclusion inattendue sur les «idées libérales» :

[...] nous espérons que le public qui a appris à lire Shakspeare dans la traduction de M. Guizot lui restera fidèle et la trouvera de tout point remise au niveau des idées libérales en littérature qui ont enfin triomphé et dont elle a si efficacement aidé le triomphe (Brochure: 3).

À l'édition de 1821, Guizot avait contribué par une longue introduction. Ce texte, De Shakspeare et de la poésie dramatique (Guizot, 1822), publié en volume séparé, se présentait à la fois comme une étude détaillée sur l'œuvre de Shakespeare, sur sa biographie et sur le contexte historique de ses pièces. Mais surtout Guizot commençait par y tracer les liens incontournables entre la littérature et la société, entre l'évolution du théâtre et les rapports entre les classes:

La littérature n'échappe point aux révolutions de l'esprit humain; elle est contrainte de le suivre dans sa marche, de se transporter sous l'horizon où il se transporte, de s'élever et de s'élargir avec les idées qui le préoccupent, de considérer enfin les questions qu'elle agite dans toute l'étendue que leur donne l'état nouveau de la pensée et de la société (Guizot 1822: 3-4).

La littérature n'est pas une entité privilégiée, une activité séparable des vicissitudes de la société ${ }^{12}$. Tout ce que Guizot aura à dire de la poésie dramatique découlera de cette position de base et l'accentuera. En republiant la traduction de Shakespeare en 1852, Guizot reprenait de très grands morceaux du texte De Shakspeare et de la poésie dramatique en l'incorporant à un ouvrage plus long et détaillé - 528 pages au lieu des $152 \mathrm{du}$ texte de 1822. De plus, l'ouvrage portait un nouveau titre: Shakespeare et son temps. Étude littéraire (Guizot 1852). Et de fait, le texte de 1852 ressemble plus à un travail d'érudition en histoire littéraire, que son prédécesseur de 1822 qui prenait délibérément l'allure d'une sorte de manifeste politique. Mais en 1852, la situation de l'auteur des deux textes avait beaucoup évolué.

En 1821, la première Restauration prenait le tournant de la réaction ${ }^{13}$. Dans le processus d'épuration qui suivit, Guizot, déjà très engagé dans l'opposition libérale, perdait sa chaire d'histoire à la Faculté des lettres. C'est ce qui l'amenait justement à la traduction de Shakespeare. En même temps qu'il se préoccupait de refondre la traduction de Shakespeare par Letourneur, Guizot s'acharnait, dans les nombreux pamphlets et livres qu'il publiait dans les mêmes années, à mettre en cause la direction adoptée par le nouveau gouvernement de Louis XVIII ${ }^{14}$. Dans une très large mesure, l'essai qu'il ajoutait en guise d'introduction à cette traduction participait aussi à cette critique politique. 
Surtout, il ne suffisait pas pour Guizot de rendre en français un équivalent linguistique aussi exact que possible des textes de Shakespeare. Traduire Shakespeare prenait pour Guizot un autre sens: il fallait aussi que cette traduction serve à faire concevoir à la société française à laquelle elle était adressée que le théâtre pouvait occuper une autre place, jouer un autre rôle dans la société que ceux qui passaient pour normaux ou naturels dans l'idée de ses concitoyens. Et, tel qu'il comprenait luimême le rôle du théâtre dans la société anglaise, une nouvelle conception du théâtre devait laisser entrevoir une nouvelle structure sociale, une autre répartition dans le statut des classes sociales, une autre distribution des droits et des pouvoirs entre ces classes: bref, une nouvelle façon de concevoir le contrat social. Faire comprendre et accepter comme vraisemblable une autre sorte de théâtre était aussi pour lui une façon de rendre pensable une autre culture politique. Pour Guizot, une traduction de Shakespeare ne saurait donc être complète sans véhiculer aussi cette intrication des structures sociales, culturelles et politiques. Il lui était donc essentiel d'expliquer en long et en large la place de Shakespeare dans la pensée anglaise et, plus généralement, le rôle et l'influence du théâtre dans la culture politique de l'Angleterre.

Mais pour que l'explication de cette influence puisse avoir quelque effet sur la société française à laquelle il adressait son texte, il fallait aussi expliquer pourquoi le théâtre en France n'avait pu atteindre le même statut. Or toute question relevant du théâtre retombait inévitablement dans la querelle entre classiques et romantiques, qui elle-même se greffait aux débats politiques du moment ${ }^{15}$. La traduction de Guizot entrait nécessairement dans cette controverse - ce que Guizot savait bien. Mais pour contribuer de façon efficace à donner au débat la dimension politique qu'il pensait lui revenir, il devait reconstituer dans ce sens l'histoire de la poésie dramatique en Angleterre. Et il était clair, pour Guizot comme pour ses lectrices et lecteurs, que l'histoire - aussi loin dans le passé qu'elle allait remonter - était mise au service d'une cause «pressante»:

On ne s'étonnera donc pas, je l'espère, si, pour connaître Shakspeare, j'éprouve moimême le besoin d'entrer un peu avant dans la nature de la poésie dramatique et dans la civilisation des peuples modernes, surtout de l'Angleterre, Si l'on n'aborde ces considérations générales, il est impossible de répondre aux idées confuses peut-être, mais actives et pressantes, qu'un tel sujet fait naître maintenant dans tous les esprits (Guizot 1822:4).

La stratégie qu'adopte Guizot dans le Shakespeare de 1822 est de montrer comment le théâtre en France a été approprié par les classes dominantes et comment cette appropriation aurait à la fois dénaturé le théâtre, l'aurait détourné de sa vocation naturelle ou tout au moins légitime, et aurait par cela même retardé - par rapport à l'Angleterre - le processus de démocratisation de la société française. Enfin, pour que telle explication ne soit pas perçue comme simplement et délibérément incitatrice, pour qu'elle ne soit pas écartée d'emblée et rangée parmi les provocations politiques courantes à l'époque, Guizot choisit de situer les origines du théâtre au rite, à la pratique religieuse. Mais en tant que protestant, il était important pour Guizot d'indiquer qu'il ne s'agissait pas d'une origine religieuse sectaire et exclusive. Il lui fallait, dans ce contexte, associer la religion à une éthique chrétienne commune. Et il fallait en même temps que ces valeurs illustrent, à la fois dans le théâtre et dans l'idéologie politique que Guizot voulait mettre en avant, des valeurs universelles et à l'abri de tout soupçon politique. Il colorait par là sa critique de connotations conservatrices: 
C'est seulement en sortant de la vie et des intérêts individuels, que l'imagination s'exalte, que l'âme s'agrandit, que les plaisirs deviennent désintéressés et les affections généreuses, que les hommes enfin peuvent se rencontrer dans ces émotions communes dont les transports font retentir le théâtre. Aussi la religion a-t-elle été partout la source et la matière primitive de l'art dramatique; il a célébré en naissant, chez les Grecs, les aventures de Bacchus, dans l'Europe moderne, les mystères du Christ (Guizot 1822, p. 6).

Mais alors qu'en Angleterre toutes les classes se retrouvaient ensemble au théâtre et partageaient à la fois émotions et leçons des spectacles, en France - et ailleurs en Europe -, le théâtre aurait été rendu le domaine d'intérêt exclusif de l'aristocratie. En Angleterre, le théâtre a pu suivre un cours que Guizot voit comme naturel: peuple et petite noblesse réunis dans une même salle, à un même spectacle, pouvait entamer le dialogue, se comprendre et comprendre en même temps que leurs intérêts n'étaient pas nécessairement contraires lors qu'il s'agissait de résister à une montée d'absolutisme, à des tentatives de saisie de pouvoir par le monarque ou à ses refus d'accorder droits ou privilèges. Pour Guizot, ce genre d'entente représentait une prolongation normale de ces origines populaires du théâtre: "La poésie dramatique n'a donc pu naître, n'est jamais née qu'au milieu du peuple» (Guizot 1822: 4).

Et Guizot insistera, comme peu d'autres - même parmi ceux qui étaient au sein de la controverse sur le renouvellement du théâtre -, sur le rôle attribué au peuple dans le théâtre de Shakespeare:

L'intervention du peuple qui porte une si lourde part du poids de l'histoire, est assurément légitime, au moins dans les représentations historiques. Elle était nécessaire à Shakspeare. Ces tableaux partiels de l'histoire privée ou populaire, placés loin derrière les grands événemens [sic], Shakspeare les attire sur le devant de la scène (Guizot, 1822: 88).

Pour Guizot, le théâtre avait une mission sociale: il devait servir à éduquer le peuple: parce qu'il représente de l'histoire, le théâtre doit faire germer en lui les idées de progrès et de civilisation. Il doit lui faire aspirer aux idéaux inscrits dans les représentations dramatiques et véhiculées par celles-ci. Mais cette mission comporte des dangers, la possibilité d'effets contraires et indésirables:

Né ainsi au milieu du peuple et pour le peuple, mais appelé à l'élever en le charmant, l'art dramatique est devenu dans tous les siècles, dans tous les pays, et par ce caractère même de sa nature, le plaisir favori des classes supérieures.

C'était sa tendance; il y a trouvé aussi son plus dangereux écueil. Plus d'une fois, se laissant séduire à cette haute fortune, il a perdu ou compromis son énergie et sa liberté (Guizot 1822: 6-7).

En s'identifiant graduellement aux intérêts des classes supérieures, l'art dramatique se corrompt et, avec ces classes supérieures qui s'isolent du reste du peuple, il en vient à perdre tout rapport à la simple humanité, à se raffiner en art hermétique obsédé par l'idée de définir le goût, le style ou la forme. Et c'est contre cela que Guizot se situe par rapport à la controverse sur le théâtre: pour lui, s'astreindre à une esthétique qui met en valeur les règles, les problèmes de forme plutôt que la représentation d'une réalité reconnaissable c'est perdre de vue le potentiel politique du théâtre. En s'identifiant graduellement aux "classes supérieures», le théâtre est devenu le domaine exclusif d'une élite oisive qui dédaigne tout contenu représentatif, historique en faveur de questions de forme: 
Ces sentiments universels, ces idées naturelles, ces relations simples, qui sont le fond de l'humanité et de la vie, s'énervent et s'altèrent dans une condition sociale toute d'exception et de privilège. Les conventions y prennent la place des réalités; les mœurs y deviennent factices et faibles (Guizot 1822: 7).

Or, selon Guizot, cet effet d'exclusion et d'aliénation n'aurait pas eu cours en Angleterre. Par contre, lorsqu'il raconte l'évolution contraire à la nature du théâtre - celle que le théâtre a suivi en France - quand il parle de cette «secousse littéraire» qui agite l'Europe, Guizot montre du doigt la France et l'Italie pour déplorer leur conservatisme irrationnel. C'est là, selon lui, la cause du bouleversement qu'ils subissent et continueront de subir: "L'Italie seule et la France, patries du classique moderne, s'étonnent du premier ébranlement donné à ces opinions qu'elles ont établies avec la rigueur de la nécessité, et soutenues avec l'orgueil de la foi» (Guizot, 1822: 118-119). Enfin, Guizot ne manque pas, ici non plus, d'évoquer explicitement les liens entre littérature et politique et de rapprocher les enjeux de la controverse littéraire des effets des récentes révolutions que ses lecteurs n'ont pas eu le temps d'oublier:

Il semble que la discussion porte un aspect menaçant, et que l'examen ne puisse sonder sans renverser. Dans cette situation on hésite comme au moment de détruire ce qu'on ne remplacera point; on a peur de se trouver sans loi, de ne rien découvrir que l'insuffisance ou l'illégitimité des principes sur lesquels on se plaisait à s'appuyer sans inquiétude (Guizot 1822: 118-119).

L'allusion était d'autant plus risquée que la véritable visée du passage - la menace sombre d'une révolution à venir - y transparaissait. Mais tout en rattachant la controverse sur le théâtre aux remous politiques du moment, Guizot s'appuyait aussi bien sur sa réputation d'historien et son expertise largement reconnue en histoire de l'Angleterre. Surtout, Guizot, on le voit aussi dans ce dernier passage, préconisait une attitude pondérée: il appelait à l'examen des positions de chacun - non pas à la confrontation. L'esprit de tolérance politique et religieuse qu'il disait fleurir sous Elisabeth et qu'il décrivait dans cette introduction devait implicitement servir de modèle à la France. Il s'agissait non seulement de comprendre Shakespeare mais aussi d'adopter les attitudes d'ouverture d'esprit et de compromis qui avaient permis à son talent de s'épanouir. Il est certain que Guizot tenait avant tout à sauvegarder les droits et libertés acquis par la Révolution ${ }^{16}$. Mais il craignait - malgré les nombreuses références au rôle du peuple qu'il soulignait partout dans l'œuvre de Shakespeare - la démagogie populaire et la violence des révolutions autant que les Bourbons.

Le double emploi sous la Restauration d'écrits a priori sans pertinence politique, et mis au service de la cause libérale ou même républicaine, était très répandu. Les traductions - tout particulièrement celles de textes anglais - étaient très usitées parce qu'elles attiraient rarement l'attention de la censure. Ainsi, si d'un côté la défaite de 1815 et l'occupation des alliés prolongeaient le ressentiment de la gauche et de la droite françaises contre l'Angleterre, d'un autre, la longue tradition de dialogues philosophiques et d'échanges d'écrits entre intellectuels français et anglais du dix-huitième siècle se prolongeait sous la Restauration et continuait à fournir des armes contre les velléités absolutistes des Bourbons et des Ultras. En même temps que la Restauration et tout au long de celle-ci, la grande popularité en France des écrivains anglais - et en particulier celle des romans historiques de Walter Scott - servait elle aussi les fins des libéraux français. 
Souvent, les traductions d'articles de journaux et de revues anglaises offraient des moyens de critiques d'autant plus subtiles et efficaces qu'elles ne critiquaient rien ouvertement. Telles traductions se contentaient de rendre publics en France des statistiques industrielles, des bilans de commerces coloniaux ou d'échanges internationaux, des annonces d'expansion du réseau de chemin de fer britannique ou de l'installation de nouvelles technologies. La comparaison n'était le plus souvent qu'impliquée. Mais elle suffisait à rappeler et à souligner l'inefficacité des gouvernements et des ministères français. Après tout, la France, comme l'Angleterre, était en principe une monarchie constitutionnelle. Mais il était évident qu'en France les choses allaient moins bien et que le style indécis et capricieux de gouvernement des Bourbons laissait la France en retard par rapport à la Grande-Bretagne. Faire innocemment l'inventaire des progrès industriels et commerciaux britanniques c'était dénoncer l'ineptie d'un régime français alourdi par des pratiques administratives désuètes et toujours encombré de dettes politiques envers ses loyaux serviteurs - eux aussi déterminés à perpétuer les structures de l'ancien régime ${ }^{17}$.

Guizot, pour sa part, mettait moins d'égards dans sa critique du gouvernement. Dans ses discours politiques comme dans ses écrits historiques, il n'hésitait pas à donner ouvertement les institutions parlementaires anglaises comme modèles que la France devait et pouvait aisément adopter ${ }^{18}$. Il demeure que le dialogue philosophique entre Anglais et Français se poursuivait et engageait maintenant une plus large proportion des deux peuples, ne se limitant plus aux élites intellectuelles et politiques. Mais chez Guizot sans doute bien plus que chez d'autres l'Angleterre devenait comme un point de repère, une base de comparaison incontournable.

\section{Section III : Shakespeare et l'histoire comparée}

Nulle époque peut-être n'est plus favorable à la fécondité de l'esprit, que ces temps où la liberté, s'ignorant elle-même, jouit naïvement de ce qu'elle possède sans s'apercevoir de ce qui lui manque, temps pleins d'ardeur, mais peu exigeans [sic], où les droits n'ont pas été définis, les pouvoirs discutés, les restrictions convenues. Le gouvernement et le public, marchant alors sans crainte et sans scrupule, chacun dans sa carrière, vivent ensemble sans s'observer avec méfiance, ne se rencontrant même que rarement (Guizot 1822: 15).

Dans le texte de 1822, présenter Shakespeare au public français se révèle très vite une bonne occasion de comparer l'évolution politique des deux nations ${ }^{19}$. Et la comparaison, dès qu'elle porte sur l'évolution politique, se joue chaque fois en faveur de l'Angleterre.

Dans ses écrits, Guizot revient si souvent à l'Angleterre que cela en vient à ressembler à un automatisme. C'est, par exemple, le cas dans ses Essais sur l'histoire de France de 1836, où il ouvre soudain, dès la préface et sans motivation apparente, cette parenthèse où il compare l'évolution politique de la France à celle de l'Angleterre:

La France n'est entrée dans la carrière de la liberté politique qu'après avoir fait des progrès immenses dans celle de la civilisation; en Angleterre un gouvernement libre est né du sein de la barbarie. C'est là le grand fait qui distingue profondément les deux peuples, qui influera longtemps encore sur leur caractère et leur destinée, et que j'ai entrepris d'expliquer (Guizot 1847: v). 
L'effet de cette intervention inattendue de l'histoire d'Angleterre dans une série d'essais ostensiblement consacrés à l'histoire de France est de faire dépendre la compréhension de celle-ci de la compréhension de celle-là. Et lorsqu'il referme la parenthèse quelque 250 pages plus loin pour relever le retard "politique» de la France relativement à l'évolution de la Grande-Bretagne, il ne fait que confirmer l'importance cachée de cette comparaison:

[...] la formation progressive de la monarchie française n'est point une œuvre politique, la lutte d'un pouvoir central contre une aristocratie qui défend et perd ses libertés: c'est une série de conquêtes, la guerre d'un prince contre d'autres princes qui défendent et perdent leurs États. Là réside la différence fondamentale qui a distingué la France de l'Angleterre, et a décidé longtemps du sort des deux pays (Guizot 1847:251) ${ }^{20}$.

Enfin, le sixième des Essais sur l'histoire de France, intitulé «Des Causes de l'établissement du gouvernement représentatif en Angleterre, » confirme, s'il le fallait, ce rôle de modèle que devait jouer l'Angleterre. Mais, à en croire ses Mémoires, les effets de cette comparaison allaient au-delà d'une compréhension uniquement théorique des différences d'évolutions ou de systèmes de gouvernement. Lorsque beaucoup plus tard, il revient sur les évènements de la Révolution de Juillet qui l'avait amené, lui et ses collègues libéraux, au pouvoir en 1830, Guizot avoue l'effet très étendu de cette idéalisation de l'Angleterre sur la politique qu'ils avaient à dessiner, sur les décisions qui en découlaient et qu'ils avaient mises en pratique:

[...] nous avions l'esprit plein de la révolution de 1688 en Angleterre, de son succès, du beau et libre gouvernement qu'elle a fondé, de la glorieuse postérité qu'elle a value à la nation anglaise. Nous ressentions l'ambition et l'espérance d'accomplir une ouvre semblable, d'assurer la grandeur avec la liberté de notre patrie, et de grandir nousmêmes dans la poursuite de ce dessein (Guizot 1858-1865: II, 19).

Ces ambitions étaient indéniablement louables, mais il reste qu'il s'agissait pour un gouvernement d'en imiter un autre, de rattraper un modèle qui, au moment où se décide ladite imitation, avait presque 150 ans d'avance. À lire un tel texte, on peut penser avoir trouvé chez Guizot une sorte de foi aveugle - comme si l'histoire de l'Angleterre était devenue pour lui une sorte d'allégorie prophétique qu'il s'agissait pour lui de remettre en scène, de rejouer au bénéfice de la France dont il avait maintenant à mener les destinées. Pourtant, la pratique telle qu'il la décrit ici s'accorde chez Guizot avec ses théories sur l'histoire et sur l'évolution politique des peuples. Et l'on retrouve encore des esquisses de ces théories jusque dans le Shakspeare de 1822 - et cela autant dans la mesure où il attribue à Shakespeare sa propre perspective historique que dans celle où cette perspective lui sert à comprendre et à expliquer Shakespeare.

Pour Guizot, la causalité en histoire est rarement directe. Comprendre les causes d'un événement donné implique selon lui la prise en considération et l'analyse d'une très longue durée. C'est ce qu'il indique de façon très brève dans une lettre de 1829 à l'un de ses amis, historien et allié politique, M. de Barante:

C’est le fait dont je suis le plus frappé dans toute notre ancienne histoire: elle est prodigieusement complexe; une foule de causes et d'éléments hétérogènes y ont concouru; aucun assez fort pour dominer seul, assez apparent pour tout éclairer (Guizot in Bardoux 1894: 52). 
Mais on voit très souvent Guizot exprimer dans ses écrits historiques cette nécessité de considérer la très longue durée. Le plus souvent il l'exprime de façon plus élaborée. Comme, par exemple, dans les Essais sur l'histoire de France:

Les causes des révolutions sont toujours plus générales qu'on ne le suppose; l'esprit le plus pénétrant et le plus vaste ne l'est jamais assez pour percer jusqu'à leur première origine, et les embrasser dans toute leur étendue. Et je ne parle pas ici de cet enchaînement nécessaire des événements qui fait qu'ils naissent constamment les uns des autres, et que le premier jour portait dans son sein l'avenir tout entier. Indépendamment de ce lien éternel et universel de tous les faits, il est vrai de dire que ces grandes vicissitudes des sociétés humaines que nous appelons des révolutions, le déplacement des pouvoirs sociaux, le bouleversement des formes de gouvernement, la chute des dynasties, datent de plus loin que ne le dit l'histoire, et proviennent de causes bien moins spéciales que celles qu'elle leur attribue communément (Guizot, $1847: 46$ ).

Il n'y a donc pas pour Guizot de raccourcis pour comprendre l'histoire. Il n'y en a surtout pas pour expliquer ce phénomène qui hante toujours les esprits de ses contemporains: la révolution. D'autant plus que les protagonistes, ceux qui croient mener les événements ou y participer, ne sauraient en comprendre toute la portée ni même entrevoir les répercussions de leurs gestes. Et Guizot reprend cette idée explicitement dans son ouvrage le mieux connu et le plus souvent traduit, son Histoire de la civilisation en Europe:

L'homme avance dans l'exécution d'un plan qu'il n'a point conçu, qu'il ne connaît même pas; il est l'ouvrier intelligent et libre d'une œuvre qui n'est pas la sienne; il ne la reconnaît, ne la comprend que plus tard, lorsqu'elle se manifeste au dehors et dans les réalités; et même alors il la comprend que très incomplètement [...] chacun cependant exécute avec intelligence et liberté, par des actes rationnels et volontaires ce dont il est chargé (Guizot 1846: 293).

Guizot était conscient de tous les avantages que représentait, en fait de procédé dramatique pour Shakespeare, cette cécité des personnages historiques. L'usage que Shakespeare en avait fait informait sans doute dans une large mesure cette perspective de Guizot. Mais lorsque qu'il en énumère les exemples dans les pièces de Shakespeare c'est à la fois en leur ajoutant une dimension religieuse et en la ramenant, pour les besoins du drame, sur le plan de l'individu, du personnage principal. C'est ce personnage central dans chacune des grandes tragédies shakespeariennes qui dès lors représente l'humanité tout entière dans l'histoire et qui dans le drame devient,

l'instrument et l'objet des décrets de la destinée qui, dans ce monde créé pour l'homme, a voulu que tout se fit $[s i c]$ par les mains de l'homme, et rien selon ses desseins. Elle emploie la volonté humaine à accomplir des intentions que l'homme n'a point eues, et le laisse marcher librement vers un but qu'il n'a pas choisi (Guizot 1822: 89).

À bien entendre l'importance de cette longue perspective dans la pensée historique de Guizot, on comprend mieux son opposition à la représentation de la traduction par l'image de la photographie. La photographie a déjà commencé à se donner et à se lire comme archive à l'époque où Guizot en fait la critique. Et c'est en tant qu'archive que la distorsion photographique menace ce qui compte le plus de la représentation pour Guizot. La photographie substitue l'instant, le moment capté à la durée réelle d'une action, d'un geste, d'une bataille, d'une vie. Et l'objectivité mécanique présumée de l'instrument photographique se transpose sur l'instantané aléatoire qu'il produit. 
C’est ce qui fait «grossir» des faits qui ne mériteraient pas autrement d'attirer tant d'attention. Au contraire, dans une compréhension fondée sur la longue durée, l'instantané devient presque insignifiant. De là la difficulté pour la photographie de servir de modèle à une traduction, à une représentation d'une œuvre qui pour Guizot renfermait tout le système d'une culture, d'une civilisation qu'il voulait faire servir de modèle politique. Tout exacte qu'elle puisse être à saisir le moment dramatique ou historique, et même lorsqu'elle le fait avec une extrême précision, la photographie ne saurait adéquatement représenter les valeurs, les idéologies, tout ce qui motive enfin ce moment pour le projeter sur la scène de l'histoire. Encore moins saurait-elle donner une idée de l'enchevêtrement d'idées régnant dans l'esprit des êtres dont elle ne fixe l'expression que d'un seul instant - si toutefois l'expression inscrite sur un visage pouvait révéler une pensée politique ou quelque perspective que ce soit sur l'histoire. À la limite la photographie ne saurait représenter qu'un fait évanescent rendu caduque d'avoir été découpé et détaché du tout qui lui confère un sens. Une traduction qui aurait escamoté la dimension temporelle d'un texte, qui en aurait aplati les motifs et les antécédents, ne pourrait jamais satisfaire Guizot. C'est ce qui rend essentielle pour lui la composition de ces longues introductions qu'il joint à ses traductions.

\section{Section IV : Traduire les faits ou traduire leur sens}

C'est un désordre grave et un grand affaiblissement chez une nation que l'oubli et le dédain de son passé. Elle peut, dans une crise révolutionnaire, se soulever contre des institutions vieillies et insuffisantes; mais quand ce travail de destruction est accompli, si elle continue à ne tenir nul compte de son histoire, si elle se persuade qu'elle a complètement rompu avec les éléments séculaires de sa civilisation, ce n'est pas la société nouvelle qu'elle fonde, c'est l'état révolutionnaire qu'elle perpétue (Guizot 1858-1865: I, 336).

À l'époque où Guizot compose ces lignes dans ses Mémoires, elles sont loin encore de connoter pour lui ce sentiment abstrait et résigné devant la répétition d'un passé qu'on aurait collectivement négligé de comprendre; sentiment que l'on y reconnaît aujourd'hui. «L'oubli et le dédain » du passé condamnent les nations, celles qui s'en rendent coupables, à la révolution perpétuelle, à la répétition cyclique de crises passées. En 1858, Guizot avait de cela une connaissance intime car il n'avait pas fait qu'écrire de l'histoire. Une dizaine d'années plus tôt, le gouvernement qu'il dirigeait en tant que premier ministre se voyait renversé par une nouvelle révolution. Tout comme une autre révolution, celle de juillet 1830, lui avait encore une fois ouvert les portes du gouvernement et l'accès au pouvoir. Mais, né en 1787 et fils de guillotiné, Guizot en avait vu d'autres.

Reste néanmoins que, dans la perspective de la très longue durée, et en considérant ce que Guizot sait déjà de ces protagonistes aveugles qui font l'histoire, le problème peut se poser de savoir pourquoi il trouve encore bon de chercher à comprendre l'histoire.

Pour Guizot, ce dilemme se résout d'abord par la recherche méthodique en histoire: il ne s'agit pas pour lui uniquement d'accumuler et de vérifier les faits mais d'en comprendre le sens, d'en extrapoler la direction, les tendances générales et souvent répétées ou reprises dans l'évolution progressive des nations. Le grand thème de Guizot est celui de la «civilisation ». C'est ce qu'indiquent par exemple les 
titres de ses ouvrages les plus étudiés, lesquels représentaient généralement la substance de ses cours. Au fil des années, Guizot modifiera les définitions qu'il donne de la civilisation, il en accentuera plus ou moins certains aspects. Mais une constante la raison - demeurera essentielle dans toutes les descriptions qu'il en donnera. La raison justifiera même le pouvoir absolu si celui-ci est considéré à partir d'une longue perspective historique.

Ainsi, dans ses Essais, ayant une fois expliqué comment Charlemagne avait établi son pouvoir par la violence, Guizot tire la leçon selon lui la plus importante: Charlemagne aura unifié l'État, créé la nation par la force. Et Guizot cherchera à aller audelà de cette contradiction évidente entre violence et raison en invoquant la justification fournie dans une plus longue échéance historique: cette violence politique était essentielle à l'unification de la nation qui aura été son ultime aboutissement:

[...] c'est de ce chaos que Charlemagne fit, pour un moment, une monarchie. Quiconque s'étonnerait de ce triomphe passager du système monarchique n'aurait compris ni ce qu'est un grand homme, ni ce que veut au fond toute société. Petite ou étendue, civilisée ou barbare, ce qu'elle cherche, ce qu'elle invoque, c'est l'empire de la raison, de la justice, le règne du pouvoir légitime. Là réside l'unique et dernier but de toute réunion d'hommes: toutes les formes de gouvernement, toutes les institutions, toutes les garanties ne sont que des moyens (Guizot 1847: 183).

Guizot donnera de nombreux exemples de ce rôle marqué de la raison dans l'évolution des États. La raison les a amenés insensiblement vers la démocratisation, vers une participation plus étendue des classes - et surtout de cette classe dont il se réclame et qu'il appelle «moyenne». Cette redistribution des pouvoirs politiques est pour lui quasiment une nécessité historique. Mais pour vérifier ses hypothèses, pour voir si en fait la raison est le principe qui, dans la réalité, règle l'évolution des états plus avancés, ce sera encore sur le modèle de l'Angleterre que Guizot s'appuiera. Si les régressions politiques de la monarchie soi-disant constitutionnelle des Bourbons parviennent souvent à mettre en doute le sentiment qu'il a que le progrès de l'État vers la démocratie est nécessaire, ce sera par l'exemple de la Grande-Bretagne que Guizot se trouvera confirmé dans sa pensée ${ }^{21}$. Le modèle anglais sert de confirmation au-delà de la théorie, dans le concret, dans l'actuel ${ }^{22}$. Guizot croit voir son interprétation de l'histoire traduite en réalité dans l'évolution et l'amenuisement progressifs des institutions démocratiques en Angleterre. L'existence même de ces institutions confirme pour lui leur nécessité historique - non seulement en Angleterre, mais aussi en France où elles sont encore loin d'être reconnues comme légitimes.

Mais l'Angleterre n'est pas un modèle sans ambivalences pour Guizot. Quand il compare leurs niveaux d'évolution politique, l'Angleterre est bien en avance sur la France comme sur les autres nations de l'Europe. Elle l'est d'abord parce qu'elle a su focaliser son énergie sur l'amélioration de la «condition extérieure et publique des hommes» :

[...] vers l'amélioration, non pas seulement de la condition matérielle, mais aussi de la condition morale; vers l'introduction de plus de justice dans la société, comme de plus de bien-être; vers le développement du droit, comme du bonheur (Guizot 1864: 9).

Mais elle n'est pas pour autant celle des deux nations à avoir atteint le plus haut degré de civilisation - et cela justement par ce même sens pratique qui tout en assurant son 
évolution politique contraint son imagination ou son intérêt pour la théorie et l'abstraction, pour la philosophie:

[...] les hommes qui [en Angleterre] semblent voués par profession au développement de l'intelligence pure [...], appartiennent à l'école philosophique qu'on peut appeler pratique; il s'inquiètent surtout des résultats immédiats et positifs; ils ne se confient ni aux élans de l'imagination, ni aux déductions de la logique: ils ont le génie du bon sens (Guizot 1864: 9).

Même dans la période de sa plus grande activité intellectuelle, dit Guizot, «la crise politique et religieuse des $\mathrm{XVI}^{\mathrm{e}}$ et $\mathrm{XVII}^{\mathrm{e}}$ siècles ", l'Angleterre n'a pas su produire une école de pensée philosophique qui ait su s'enraciner ailleurs en Europe, ou qui ait élargi «l'horizon de l'esprit humain» (Guizot 1864: I, 10) 23. $^{23}$

Dans la structure de la pensée historique de Guizot, l'Angleterre, toute idéalisée qu'elle soit, reste quand même un fait à part. Un fait qui peut servir d'exemple et qui renforce cette hypothèse d'un progrès politique généralisé de la civilisation humaine. Mais en même temps elle reste un exemple différent dans sa nature et du point de vue de cette valorisation excessive des faits par rapports aux idées: l'Angleterre reste inférieure à ce que la France, dans l'esprit de Guizot, est capable d'atteindre en fait de civilisation.

Car il faut compter que les faits n'ont pas toujours un statut cognitif très assuré dans les écrits de Guizot. Si Guizot adopte le discours scientifique qui accorde aux «faits» une position dominante, s'il semble défendre et adhérer à la méthode qu'il nomme "philosophique», c'est pour très vite la qualifier, et surtout l'adapter à sa conception du «fait» dans l'histoire. Par ailleurs, pour bien entendre son adhésion, il faut savoir que le mouvement philosophique au travers duquel il se réfere à cette méthode, au culte du fait, a toujours eu pour lui un statut ambivalent. Cela probablement parce que cette "philosophie», celle des Lumières, se rattache dans ses écrits à deux sortes de suites. Sans doute, la philosophie du dix-huitième siècle a-t-elle donné voix et raison aux droits de l'homme qui constituent la base de son idéologie libérale. Mais elle a aussi servi - par abus - la démagogie révolutionnaire et de là la terreur qui a perverti et diffamé cette idéologie. Lorsque, dans son cours sur l'Histoire de la civilisation en France, Guizot proclame son adhésion à la méthode philosophique, à la mise en valeur du fait, de l'objectivité, il le fait d'abord comme si c'était une narration faite de l'extérieur, comme s'il rapportait une série d'événements qu'il n'avait fait qu'observer :

Quel est l'esprit qui prévaut aujourd'hui dans l'ordre intellectuel, dans la recherche de la vérité, quel qu'en soit l'objet? un esprit de rigueur, de prudence, de réserve, l'esprit scientifique, la méthode philosophique. Elle observe soigneusement les faits, et ne se permet les généralisations que lentement, progressivement, à mesure que les faits sont connus. Cet esprit domine évidemment depuis plus d'un demi-siècle, dans les sciences qui s'occupent du monde matériel; il a fait leur progrès et leur gloire. Il tend aujourd'hui à pénétrer de plus en plus les sciences du monde moral, dans la politique, l'histoire, la philosophie. Partout la méthode scientifique s'étend et s'affermit; partout on sent la nécessité de prendre les faits pour base et pour règle; on est persuadé qu'ils sont la matière de la science, qu'aucune idée générale ne peut avoir de valeur réelle si elle n'est sortie du sein des faits, si elle ne s'en nourrit constamment à mesure qu'elle grandit. Les faits sont maintenant, dans l'ordre intellectuel, la puissance en crédit (Guizot 1864: I, 22-23). 
Guizot va très vite prendre ses distances; il va se défendre de ce culte du factuel. Pour lui, le fait ne saurait se saisir littéralement, simplement. Certes, il faut se fonder en histoire sur des faits et non des illusions. Mais, en histoire, le fait ne peut pour cela se limiter à des événements, à des actes concrets susceptibles d'être inscrits ou archivés dans quelque registre comme les batailles, les guerres ou les insurrections. Le «fait» en histoire doit - entre autres - pouvoir aussi admettre, être apte à comprendre et à retracer, avec les faits matériels, l'évolution d'abstractions, d'idées. L'historien devra aussi pouvoir compter comme factuelle - comme historique -l'influence des idées sur les événements, sur les agissements d'êtres humains qui subissent ces influences. C'est donc le rôle, l'influence incontournables des idées pures dans l'histoire que Guizot défend dans la suite immédiate de ce passage:

Dans l'ordre réel, dans le monde social, dans le gouvernement, dans l'administration, l'économie politique, une autre direction se manifeste; là prévaut l'empire des idées, du raisonnement, des principes généraux, de ce qu'on appelle les théories (Guizot 1864: I, 23).

Cet élargissement du sens et du statut du fait que Guizot préconise ici, par rapport à un discours scientifique plus univoque que le sien, s'exprime de façon plus directe et plus élaborée dans un cours antérieur de Guizot, son cours sur l'Histoire de la civilisation en Europe:

Depuis quelque temps on parle beaucoup, et avec raison, de la nécessité de renfermer l'histoire dans les faits, de la nécessité de raconter: rien de plus vrai; mais il y a bien plus de faits à raconter, et des faits bien plus divers, qu'on n'est peut-être tenté de le croire au premier moment; il y a des faits matériels, visibles, comme les batailles, les guerres, les actes officiels des gouvernements; il y a des faits moraux, cachés, qui n'en sont pas moins réels; il y a des faits individuels qui ont un nom propre; il y a des faits généraux, sans nom, auxquels il est impossible d'assigner une date précise, qu'il est impossible de renfermer dans des limites rigoureuses et qui n'en sont pas moins des faits comme d'autres, des faits historiques qu'on ne peut exclure de l'histoire sans la mutiler (Guizot 1840: 16).

L'ambivalence de Guizot envers l'Angleterre et sa culture du sens pratique qui tend à marginaliser l'abstraction n'est pas sans analogie avec la distance qu'il maintient envers la méthode philosophique. Et l'on peut lire, dans le contexte du travail de traduction de Guizot - quand d'abord il traduit Gibbon - que c'est en traduisant l'historien anglais et en travaillant les discours savants désormais greffés sur son œuvre que s'est fait ressentir et exprimé chez Guizot ce besoin d'élaborer la notion du «fait» en histoire.

C'est ce qu'il paraît raconter dans une sorte d'anecdote autobiographique au début de sa "Préface de l'Éditeur» qui accompagne sa traduction de l'Histoire de la décadence et de la chute de l'Empire romain. Ayant lui-même relevé un grand nombre d'erreurs de détails chez Gibbon, Guizot se voyait sur le point de dénoncer le grand historien trouvé fautif quand il s'aperçut que cette attention prêtée aux erreurs de détail le distrayait de voir toute l'ampleur du travail, toute l'érudition de Gibbon investie dans cette œuvre. Non pas qu'ayant évité l'erreur Guizot se serait permis de négliger la vérification des faits. Néanmoins Guizot comprenait qu'il n'avait que de peu évité un écueil que beaucoup d'autres n'avaient su voir : à force de se préoccuper de faits individuels, de détails de plus en plus pointilleux, on oublie de les relier, de les interpréter: 
[...] et un grand nombre d'autres écrivains français et étrangers, en ont examiné toutes les parties [des références de Gibbon]: ils se sont enfoncés au milieu des décombres pour y chercher des faits, des renseignements, des détails, des dates; et à l'aide d'une érudition plus ou moins étendue, d'une critique plus ou moins éclairée, ils ont en quelque sorte rassemblé et arrangé de nouveau tous ces matériaux épars, leurs travaux sont d'une incontestable utilité, et je n'ai garde de vouloir en diminuer le mérite; mais en s'y enfonçant, ils s'y sont quelquefois ensevelis [...] en s'occupant de la recherche des faits ils ont négligé l'ensemble des idées, ils ont fouillé et éclairé les ruines, sans relever le monument (Guizot 1828: 2-3).

Et tel que Guizot entendait la pensée de son temps, cette tendance à survaloriser les faits - et de là à accumuler sans justification ou à débattre inlassablement les moindres détails -, cette tendance se voyait exacerbée par un discours scientifique qui de plus tendait à confondre factualité et objectivité. Parce qu'il entrevoyait les risques que représentait cette conjoncture pour l'histoire, Guizot cherchait constamment dans ses écrits et encore plus souvent dans ses cours - à mieux définir le travail de l'historien en déterminant nettement le contenu et la méthode en histoire et en les différenciant, comme on a vu, des discours scientifiques dominants. Pour Guizot, la tâche de l'historien était de présenter autre chose que les seuls faits - même si ceuxci avaient passé par les plus rigoureuses vérifications. De cette tâche, Guizot donne la meilleure description - mais en forme négative. La tâche de l'historien, selon lui, c'est justement de fournir ce que ces historiens, qui s'étaient laissé «enfoncer» dans la vérification des faits de Gibbon, n'avaient pas su trouver:

[...] ces vues générales qui nous aident à embrasser d'un coup d'œil une grande étendue de pays, une longue série de siècles, [...] ces grandes vues enfin qui constituent la partie philosophique de l'histoire, et sans lesquelles elle n'est plus qu'un amas de faits incohérens [sic], sans résultat comme sans liaison (Guizot 1828:3).

Pour Guizot, l'histoire, comme la traduction, est aussi un travail d'interprétation - ne fut-ce que parce qu'elle implique nécessairement à chaque étape et sur tous ses plans une sélection parmi les faits, parmi les périodes, les personnages ou les événements.

\section{Section V : Faire l'histoire des faits}

Une conviction n'entre point dans l'intelligence humaine si l'intelligence ne lui ouvre la porte; il faut qu'elle se fasse accepter. De quelque manière qu'elle se présente, quel que soit le nom qu'elle invoque, la raison y regarde; et si la croyance pénètre, c'est qu'elle est acceptée par la raison (Guizot 1840: 102).

En fondant en 1827, mes amis et moi, l'un des principaux recueils périodiques de ce temps, la Revue française, nous lui donnâmes pour épigraphe ce vers d'Ovide:

Et quod nunc ratio est, impetus ante fuit.

"Ce qui est maintenant de la raison a été d'abord un élan passionné.»

Nous exprimions ainsi avec vérité l'esprit dominant autour de nous, et notre propre disposition (Guizot 1858-1867: I. 323).

Guizot savait que, comparée aux sciences matérielles, l'histoire ne pouvait encore à cette époque opposer à la marée scientiste qu'une très faible résistance. En tant que science émergente, l'histoire devait se sentir pressée à se conformer, à imiter dans leur démarche les sciences naturelles ${ }^{2}$. L'histoire avait encore à se définir, à se représenter comme autre chose qu'une narration qui n'intéresse que parce qu'elle raconte aux 
êtres humains ce que d'autres êtres humains ont fait. Mais, pour avoir longtemps fréquenté les historiens, Guizot comprenait en même temps l'inanité des doctrines scientistes quand celles-ci revenaient à compter l'accumulation et la vérification scrupuleuses des faits comme l'acquittement de tout devoir scientifique. Pour Guizot, les faits pouvaient peut-être se considérer neutres ailleurs - quelque part, dans quelque conception positiviste du monde - mais pas en histoire. Telle qu'il l'entendait, l'histoire se faisait autrement. Car si jusque-là, dans les discours des historiens, les mêmes faits pouvaient servir toutes les causes, soutenir toutes les idéologies, c'est encore parce que l'histoire avait pour valeur fondamentale l'interprétation des événements. Et, pour ses étudiants, Guizot ne cessait de démontrer la nécessité d'opposer aux pressions du scientisme, le rôle incontournable de l'interprétation dans l'exposé des faits en histoire. C'est ce qui se lit très clairement dans ce passage de ses Essais, où, ayant analysé sur plusieurs pages comment les événements d'une période longue de cinq siècles ont été représentés par différents historiens, chacun de tendance politique différente, chacun selon des intérêts historiques déterminants et contradictoires, Guizot tire cette conclusion en fin de démonstration:

Je pourrais poursuivre et trouver, parmi nous aussi, matière à de tels rapprochements; mais ceux-là me suffisent pour faire voir par quelle cause l'époque dont je viens de traiter a été, selon moi, si complètement et si diversement méconnue. Elle s'est prêtée à toutes les vicissitudes de la société, à tous les besoins de l'esprit de parti, toutes les hypothèses de la science; elle a fourni des armes à tous les systèmes, à tous les pouvoirs, à la liberté comme à l'aristocratie, à l'aristocratie comme à la royauté. Pourquoi? parce qu'elle portait dans son sein toutes choses, la liberté, la royauté, le privilège; et toutes choses dans un état d'inconsistance et de confusion qui a permis à chaque siècle, à chaque parti, à chaque homme d'y voir tout ce qui lui convenait. La simultanéité déréglée des systèmes d'institutions les plus divers, des tendances politiques les plus contraires, est donc le seul caractère qu'on lui puisse assigner (Guizot 1847: 238).

Dans le contexte des conflits idéologiques dont est saturée la période de ces écrits de Guizot, le soupçon quant au statut des faits s'inscrit en profondeur. La controverse politique qui caractérise la première moitié du dix-neuvième siècle en France en venait à s'installer au centre de tous les débats, de toutes les discussions intellectuelles. Elle parvenait à tellement distraire de l'objet du savoir en soi qu'elle en arrivait presque à s'y substituer. Réagissant à cette conjoncture, les hommes de science s'évertuaient à prêcher l'objectivité. Mais Guizot comprenait qu'en termes absolus le genre d'objectivité qu'ils visaient ne pouvait être qu'illusoire en histoire. L'appareil idéologique qui entre dans la détermination de ce qui constitue un fait ou un événement ne pouvait être dissipé par de telles incantations. Surtout que les mots d'ordre de la philosophie de la science de l'époque avaient eux-mêmes leurs origines, et n'étaient d'abord applicables qu'aux «sciences qui s'occupent du monde matériel» (Guizot 1864: I, 22). Et, à bien lire ce texte de Guizot, il s'agissait aussi pour lui de faire comprendre, par exemple, que les «époques » historiques n'ont pas existé comme telles, dans la nature, comme autant de spécimens végétaux. Clairement, le discours scientiste ne pouvait s'appliquer à l'histoire telle que Guizot entendait son rôle. La part de l'interprétation - et donc de l'idéologie - en était, selon lui, inaliénable. Une des fonctions importantes de l'histoire chez Guizot consiste justement à retracer l'évolution de ce qu'il nomme plus haut «conviction » ou «élan passionné » à une idéologie, à une croyance raisonnée. Au contraire, en cherchant à créer des entités artificielles, discrètes et étan- 
ches pour ensuite les étiqueter comme «faits» ou "événements», on ne ferait que dénaturer l'histoire, en exclure arbitrairement ce qui motive les croyances ou provoque les événements. En cherchant à contenir une invasion appréhendée de la politique voire une substitution éventuelle de l'idéologie au savoir - la science en arrivait à vouloir se détacher de l'histoire. Ces idéaux scientistes ne pouvaient dès lors s'adopter en histoire sans contredire ce que Guizot comptait parmi les devoirs les plus importants du métier d'historien. Et ceux parmi les historiens qui, voulant imiter les sciences, se seraient entêtés à vouloir isoler les faits au lieu de tenter de les relier, de les resituer dans leurs contextes, s'exposaient, selon Guizot, à un risque d'un autre ordre: celui de séparer leur savoir de la réalité:

[...] si la science, en procédant du dehors au dedans, selon la méthode qui lui est propre, oubliait que ce n'est point là la méthode primitive et féconde, que les faits en euxmêmes subsistent et se développent dans un autre ordre que celui où elle les voit, elle pourrait arriver à oublier que les faits la précèdent, à méconnaître le fond même des choses, à s'éblouir d'elle-même, à se prendre, en quelque sorte, pour la réalité, et à n'être bientôt plus qu'une combinaison d'apparences et de termes, aussi vaine, aussi trompeuse que les hypothèses et les déductions de la méthode contraire (Guizot 1864 : I, 34-35).

La méthode scientifique joue indiscutablement un rôle central dans la façon dont Guizot conçoit son historiographie. Guizot ne cherche pas à discréditer la méthode mais à en élargir les définitions afin d'y constituer une part pour le travail de l'historien qui ne soit pas imitation aveugle des sciences naturelles. Mais, plus généralement, toute méthode n'a pour lui que des liens contingents avec ce à quoi elle s'applique. Et Guizot se méfie du statut exalté auquel ses contemporains ont élevé la méthode. La méthode ne doit pas distraire de l'objet du savoir ou se substituer à cette part de réalité qu'une science interroge. Un savoir qui s'écarte de la réalité telle qu'elle est cesse d'être un savoir selon Guizot. Et la réalité dont l'histoire a à s'occuper est le plus souvent une réalité vécue collectivement, impliquant les vies d'une tribu, d'une race, d'une nation. Guizot, on l'a vu, avait particulièrement insisté sur la nature communautaire du théâtre, sur les effets d'effacement de l'individu une fois situé en collectivité.

Mais le besoin de se référer à un savoir collectif s'applique plus largement dans l'épistémologie de Guizot. En fait, Guizot accorde plus de valeur au savoir collectif, à un certain «bon sens» de la collectivité, qu'aux sciences pures qui, telles qu'il les conçoit, auraient plutôt tendance à s'isoler, à se tenir à l'abri de l'influence des foules. Et cette prédilection s'exprime dans d'autres contextes que celui du théâtre ou du rituel religieux.

Guizot explique cette préférence dans son cours sur l'Histoire de la civilisation en Europe, lorsqu'il s'agit de définir le terme "civilisation", terme qui joue un rôle central dans son historiographie. Et l'explication de Guizot se transforme en narration presque en théorie de l'évolution de la langue - alors qu'il décrit ce qu'il nomme un processus d'évolution «naturelle». C'est ce processus qui garantit à la fois la stabilité et la précision du sens du terme:

C'est le sens de ce mot, son sens général, humain, populaire, qu’il faut étudier. Il y a presque toujours dans l'acception usuelle des termes les plus généraux, plus de vérité que dans les définitions en apparence plus précises et plus rigoureuses de la science. C'est le bon sens qui donne aux mots leur signification commune, et le bon sens est le génie de l'humanité. La signification commune d'un mot se forme successivement en 
présence des faits; à mesure qu'un fait se présente, qui paraît rentrer dans le sens d'un terme connu, on l'y reçoit, pour ainsi dire, naturellement; le sens du terme s'étend, s'élargit, et peu à peu les divers faits, les diverses idées que, en vertu de la nature des choses mêmes, les hommes doivent rallier sous ce mot; s'y rallient en effet (Guizot 1846: 18).

Et Guizot fait immédiatement suivre cette narration par celle du procédé de nomination dans le monde de la science, laquelle s'inscrit en contraste très marqué par rapport à la précédente :

[...] lorsque le sens d'un mot, au contraire, est déterminé par la science, cette détermination, ouvrage d'un seul ou d'un petit nombre d'individus, a lieu sous l'empire de quelque fait particulier qui a frappé leur esprit. Ainsi les définitions scientifiques sont, en général plus étroites, et, par cela seul, beaucoup moins vraies au fond que le sens populaire des termes (Guizot 1840: 18-19).

Ce qui dévalorise la terminologie scientifique aux yeux de Guizot, ce qui la rend «moins vraie », c'est l'étroitesse du procédé par lequel elle se constitue. Elle se forme dans des circonstances restreintes, exclusives, n'impliquant la participation que d'un petit nombre d'individus. Et l'effet de cette opposition ainsi marquée est de souligner l'arbitraire dans le processus de nomination scientifique, tout en l'offusquant dans celui de la constitution d'une terminologie «populaire». Et tout se raconte enfin comme si l'accord collectif sur le sens, l'adoption communautaire du terme parvenait presque à effacer cet arbitraire en s'y substituant à la fois comme convention et comme motivation du signe en soi.

Cela dit, Guizot ne cherche à tromper personne sur la nature du signe. Et ayant rédigé un Dictionnaire des synonymes, il connaît trop bien la langue pour s'y tromper lui-même. Néanmoins, son insistance ici met en relief ce souci - qui est chez lui constant - de la collectivité et de la considération qu'il convient de lui accorder. Chez Guizot, cette considération s'impose particulièrement lorsque quelque collectivité en vient à confronter l'autorité arbitraire - celle d'un prince ou d'un groupe restreint ou exclusif d'individus. Et cette considération doit, selon Guizot, s'appliquer aussi bien dans le domaine politique que dans celui de la représentation historique. On a pu le constater dans son appréciation de la présence du peuple dans les drames historiques de Shakespeare.

Mais Guizot en avait exprimé le principe plusieurs années auparavant, en 1816, dans un écrit sur l'art. Dans son Essai sur les limites qui séparent et les liens qui unissent les beaux-arts ${ }^{25}$, Guizot comparait d'abord l'efficacité de représentation relative de la sculpture et de la peinture. Et en fin de comparaison, Guizot accordait sa préférence à la sculpture lorsqu'il s'agissait de représenter des figures en état de repos mais à la peinture dès qu'il fallait représenter l'action. Et cette représentation de l'action l'amenait comme naturellement à la peinture historique, à la tâche du "peintre d'histoire» :

[...] il est appelé, par la richesse et la chaleur de son art, à nous offrir ce que la nature a de plus animé et de plus riche, l'activité des hommes s'exerçant en tout sens selon les situations où ils sont placés et les passions qui les possèdent.

Cette activité est donc le seul véritable domaine du peintre, et le nom seul de peintre d'histoire indique tout ce qu'embrasse ce domaine. C'est l'histoire en effet, cette immense série d'actions, qu'il doit faire vivre et nous retracer (Guizot 1995: 42). 
Il s'avère très vite que le portrait historique n'intéresse pas Guizot. C'est en histoire la représentation d'actions collectives qui retient surtout son attention. C'est sur cela que Guizot exprime, en termes de représentation esthétique, des priorités analogues à celles qu'il manifestait en épistémologie:

Mais dès que l'action embrasse plusieurs figures, la part qu'y prend chacune d'elle détermine sa place et son attitude; en cessant d'être isolée, elle perd le droit d'être représentée et considérée uniquement pour elle-même; c'est sur l'action que l'artiste doit appeler les regards, et non plus sur les acteurs; il doit sacrifier ce qui, dans chaque pose particulière, pourrait retenir trop longtemps l'attention et la détourner de l'ensemble, fût-ce même aux dépens de chaque figure. Il ne cherchera donc plus principalement à développer de la manière la plus avantageuse les formes de ses personnages; il ne les posera plus selon son choix; il les placera dans la situation où ils doivent se trouver en concourant à une action dont chacun d'eux n'est qu'une partie (Guizot 1995: 44).

La critique de la distorsion associée à l'excès de précision en traduction - à la traduction qui prendrait la photographie pour modèle - vient s'appliquer ici plus généralement à la représentation de l'histoire. Dans la perspective d'une très longue durée qui oriente son historiographie, Guizot propose de laisser de côté les figures individuelles afin de mieux souligner la direction, le mouvement général dans lequel l'histoire s'engage - le plus souvent à l'insu de ce qui la font. Attirer l'attention sur les individus correspondrait ainsi à la distorsion occasionnée par la volonté d'imiter la précision photographique en traduction. Dans les deux cas, il s'agit pour Guizot de représentations qui s'inscrivent inévitablement dans une perspective idéologique élargie.

Pour Guizot, la traduction de Shakespeare ne pouvait se limiter aux textes des drames et des poésies; il voyait dans cette traduction l'occasion de relever les conflits sociaux et politiques internes qui rendaient le drame shakespearien inconcevable et presque impossible à mettre en scène en France. Pour cela, il lui avait fallu faire passer dans sa biographie de Shakespeare à la fois l'histoire comparée du théâtre en France et en Angleterre et celle des institutions politiques par lesquelles ce théâtre se maintenait. Les détails que Guizot choisissait de narrer en retraçant ces évolutions parallèles ne devaient que souligner ces différences - non pas attirer sur elles-mêmes une attention indue. Et l'on peut entrevoir en cela à quel point Guizot calculait l'effet que ces détails devaient avoir sur le public qu'il visait. Mais cette subordination des détails au tout dans ces deux formes de représentation rejoue sur un autre plan cette notion centrale chez Guizot - en histoire comme dans l'évaluation du savoir - de la priorité à accorder à ce qui émane d'un mouvement collectif.

Il ne pouvait donc s'agir pour Guizot de manquer de fidélité à l'original en le traduisant - d'en manipuler ou d'en déformer les détails pour en rendre la version vraisemblable au lieu de véritable. Il s'agissait au contraire de rester fidèle à l'original en reconstituant son cadre, en élargissant autant que possible le contexte historique. Ceux de la société qui lisaient cette version pouvaient ainsi retrouver dans cette histoire élargie ce qu'ils avaient en commun avec la société d'où émanait l'original et pouvaient dès lors comprendre combien de leurs passés étaient partagés, où et comment ils se séparaient. Ils étaient aptes dès lors à mieux se situer dans une histoire qui leur était commune et devaient mieux entrevoir les moyens de la changer. 


\section{NOTES}

1. François Guizot, sur «Wallstein, tragédie en cinq actes et en vers, précédée de quelques réflexions sur le Théatre allemand et suivie de notes historiques, par Benjamin Constant de Rebecque», compte rendu publié d'abord en cinq parties dans Le Publiciste du 14 février 1809, et republié in Le Temps passé: Mélanges de critique littéraire et de morale. Paris: Perrin, 1887, p. [204]-205. L’orthographe original - par exemple ici «Shakspeare» - sera maintenue dans toutes les citations. Wallenstein devient Wallstein dans la traduction de Constant; voir sur cette traduction la longue introduction de Constant, "Quelques réflexions sur la tragédie de Wallstein et sur le théâtre allemand» in Benjamin Constant, Euvres complètes, vol. III, 2, Tübingen: Max Niemeyer Verlag, 1995. Guizot commente ces réflexions dans ce compte rendu.

2. Voir, entre autres, l'édition de Dean Milman, republiée par William Smith, Philadelphie, John D. Morris \& Company. La préface de cette édition justifie en termes très élogieux l'inclusion des commentaires et notes de Guizot. La traduction de l'œuvre de Gibbon par Guizot est encore aujourd'hui la principale version française de référence. Guizot avait aussi traduit plusieurs autres textes. Voir dans Broglie, 1990, p. 519, la liste des «éditions et traductions» de Guizot.

3. C'est ce choix de stratégie didactique qui fait qu'on ne peut presque jamais donner de courtes citations d'un texte de Guizot à l'appui de la démonstration de l'une de ses prises de position ou de l'un de ses concepts. L'élaboration en détail, l'explicitation des liens entre les détails apparemment insignifiants qui sont des constantes du style de Guizot font que les citations courtes prennent l'aspect de troncatures.

4. Voir, plus loin, les passages cités ici de son Essai sur les limites qui séparent et les liens qui unissent les beaux-arts, La Rochelle: Rumeur des Âges, 1995 (1816). Il ne saurait faire de doute que Guizot prend très au sérieux cette mission pédagogique. Cela se verra amplement confirmé, plusieurs années après sa traduction de Shakespeare, quand, sous la Monarchie de Juillet, Guizot sera nommé ministre de l'Éducation et entreprendra, au prix d'énormes efforts personnels, de dynamiser et d'élargir le réseau national d'écoles publiques.

5. Voir aussi Susan Bassnett et André Lefevere, Translation, History and Culture, Londres: Pinter, 1990; Theo Hermans, Translation in Systems: Descriptive and System-Oriented Approaches Explained, Manchester: St. Jerome, 1999; et Maria Tymoczko et Edwin Gentzler, Translation and Power, Amherst et Boston: University of Massachusetts Press, 2002.

6. Cf. Maria Tymoczko, «Translation and Political Engagement: Activism, Social Change and the Role of Translation in Geopolitical Shifts,» dans The Translator, vol. 6, no.1 (2000), p. $24:$ « [...], translators must make choices, selecting aspects or parts of a text to transpose and emphasize. Such choices in turn serve to create representations of their source texts, representations that are also partial. This partiality is not merely a defect, a lack, or an absence in a translation - it is also an aspect that makes the act of translation partisan: engaged and committed, either implicitly or explicitly. Indeed partiality is what differentiates translations of the same or similar works, making them flexible and diverse, enabling them to participate in the dialectic of power, the ongoing process of political discourse, and strategies for social change. Such representations and commitments are apparent from analyses of translators' choices word-by-word, page-by-page, and text-by-text, and they are also often demonstrable in the paratextual materials that surround translations, including introductions, footnotes, reviews, literary criticism and so forth. The very words associated with politics and ideology emphasized here suggest the nexus of metonymy and engagement in the activity of translation, indicating that the partial nature of translations is what makes them political.»

7. Brochure publicitaire pour la réédition de 1852 de la traduction de Shakespeare par François Guizot, p. 3. (italiques ajoutées). Cette brochure, retrouvée à la Bibliothèque de l'Arsenal, s'y trouve reliée dans un volume comportant des textes de discours prononcés par Guizot en tant que directeur de l'Académie française. Toute référence à ce texte sera dorénavant indiquée dans les notes par l'abréviation: «Brochure» suivie de la page.

8. London Literary Gazette, 30 mars 1822: "Among the recent novelties of the Paris Drama, endeavours have been making [sic] to introduce the Romantic taste, which begins to encroach on French literature in spite or all the dissertations which have been written in favour of the Classic taste. A little piece brought out at the Porte St. Martin [...] is intended to ridicule the romantic style in the person of Monsieur Pathos, a disciple of Lord Byron and Walter Scott. But this feeble raillery has no effect in deterring the Parisians from reading Scott's novels and Byron's poems: the new English school daily gains ground in France, and a new translation of Shakspeare [sic] has lately been produced with some success. » Voir aussi M. Moraud, Le Romantisme français en Angleterre..., 
p. 137. Selon Moraud, l'enthousiasme de Stendhal amenait régulièrement les éditeurs anglais du journal à couper les trop nombreuses références politiques afin d'éviter les complications qu'elles pouvaient attirer au journal.

9. En 1851, Guizot, désillusionné par les défaites politiques qu’il a essuyées avec et depuis la révolution de février1848, soutient toujours les idéaux libéraux qu'il a représentés à l'Assemblée et au gouvernement, mais il ne se considère plus comme acteur éventuel dans la lutte pour les faire prévaloir politiquement.

10. Grand Dictionnaire universel du XIX $X^{e}$ Siècle, article «Traduction».

11. En dehors de ses revenus en tant que ministre, simple membre de l'Assemblée, ou professeur d'histoire, Guizot dépendait exclusivement de ses publications pour vivre. De la révolution de février 1848 à la fin de sa vie, il n'aura plus que ses droits d'auteur comme revenu et il devra appliquer sans doute la même attention à la représentation publicitaire de ses écrits qu'il prêtait à l'exactitude de leur contenu. Voir sur ceci, Gabriel de Broglie, Guizot, Paris: Perrin, 1990, et Pierre Rosanvallon, Le moment Guizot, Paris: Gallimard, 1985, p. [379]-389.

12. Sur le statut de la littérature dans la société à l'époque de Guizot, voir Jean Starobinski, «Gibbon et la défense de l'érudition dans Le Statut de la littérature: Mélanges offerts à Paul Bénichou», Genève: Droz, 1982, p. 205-227. Sous ce rapport de l'inséparabilité de la littérature de la société, la prise de position de Guizot - théoricien du libéralisme - rappelle étrangement celle du théoricien marxiste Georg Lukacs. Voir de Lukacs Le Roman historique, Paris: Payot, 1965, et Balzac et le réalisme français, Paris: Maspero, 1969.

13. Sur la Restauration et sur le rôle de Guizot dans cette période, voir, de Guizot, les Mémoires pour servir à l'histoire de mon temps (8 vol.), Paris : Michel Lévy, 1858-1867, et Emmanuel de Warsquiel et Benoît Yvert, Histoire de la Restauration, Paris: Perrin (collection Tempus), 2002 (1996).

14. Dès qu'il est exclu du gouvernement de Louis XVIII, Guizot se retrouve dans l'opposition. À part l'édition et la traduction de Shakespeare, il rédige et publie plusieurs essais et livres politiques dont quelques-uns ont été republiés ces dernières années. En voici quelques titres: Des Moyens de gouvernement et d'opposition dans l'état actuel de la France (1821); Des Conspirations et de la justice politique (1821); De la peine de mort en matière politique (1822); Essais sur l'histoire de France (1823).

15. Voir plus haut la note 8 en référence au compte rendu de Stendhal. Il est à noter que le texte de Guizot précède le Racine et Shakespeare de Stendhal, plus souvent cité comme l'un des points tournants de cette querelle qui culminera en 1830 avec la bataille d'Hernani.

16. C'est justement autour de ces acquis de la Révolution que Guizot dans ses Mémoires, définit le programme politique des "doctrinaires», faction libérale dont il était considéré le porte-parole: «Les doctrinaires [...] se défendirent à la fois et du retour aux maximes de l'ancien régime, et de l'adhésion, même purement spéculative, aux principes révolutionnaires. En acceptant franchement la nouvelle société française telle que toute notre histoire, et non pas seulement 1789, l'a faite, ils entreprirent de fonder son gouvernement sur des bases rationnelles et pourtant tout autres que les théories au nom desquelles on avait détruit l'ancienne société, ou les maximes incohérentes qu'on essayait d'invoquer pour la reconstruire. Appelés tour à tour à combattre et à défendre la Révolution, ils se placèrent, dès l'abord et hardiment, dans l'ordre intellectuel, opposant des principes à des principes, faisant appel non seulement à l'expérience mais aussi à la raison, affirmant des droits au lieu de n'alléguer que des intérêts, et demandant à la France, non pas de confesser qu'elle n'avait fait que le mal, ni de se déclarer impuissante pour le bien, mais de sortir du chaos où elle s'était plongée et de relever la tête vers le ciel pour y retrouver la lumière.» François Guizot, Mémoires pour servir à l'histoire de mon temps (8 vol.), Paris: Michel Lévy, 1858-1865, vol. I, p. 157158. Voir aussi Pierre Rosanvallon, Le Moment Guizot, Paris: Gallimard, 1985, p. 26-31.

17. Dans ses premières années de publication - et jusqu'à la direction d'Amédée Pichot qui en changea l'orientation - La Revue britannique pratiquait ce genre de critique implicite. Les opinions politiques des rédacteurs n'y étaient que rarement ou jamais exprimées. La moindre accumulation de statistiques, la traduction d'articles les rapportant ou faisant état de faits matériaux décrivant les avances prises par les entreprises ou les diverses administrations britanniques suffisaient pour constituer dans cette revue un dossier d'accusation contre l'administration des Bourbons. Sur l'histoire de cette revue, voir Kathleen Jones, La Revue britannique: Son histoire et son action littéraire (1825-1840), Paris: Droz, 1939.

18. Dans le Shakspeare de 1822, il dénonce indirectement le gouvernement de la Restauration pour sa faiblesse et son ineptie dans ses tentatives de censurer l'opposition politique. Il le fait en accentuant le degré de tolérance des oppositions et des controverses sous Elisabeth, à l'époque de Shakespeare: «L'ardeur poétique, l'âpreté religieuse, les querelles littéraires, les controverses théologiques, le goût 
des fêtes, le fanatisme des austérités, la philosophie, la critique, les sermons, les pamphlets, les épigrammes, tout se produisait, tout se rencontrait, se croisait ; et dans ce conflit naturel et bizarre se formaient la puissance de l'opinion, le sentiment et l'habitude de la liberté; forces brillantes à leur première apparition et imposantes dans leur progrès, dont les prémices appartiennent au gouvernement habile qui sait les employer mais dont la maturité menace le gouvernement imprudent qui voudra les asservir. L'élan qui a fait la gloire d'un règne peut devenir bientôt celui qui précipite les peuples dans les révolutions.» Shakspeare, 1822, p. 18.

19. Margaret Gilman résumait un peu de la même façon les stratégies des romantiques français quant à Shakespeare et à son rôle dans le renouvellement du théâtre qu'ils préconisaient: « [...] the enthusiasm of the romanticisits was not so much for Shakespeare as a whole as for the exemplification of their own ideas and theories which they found in his works. He was a symbol rather than a model. » Othello in French, Paris: Champion, 1925, p. 118-119.

20. On trouve une réflexion analogue dans ses Mémoires, vol. I, p. 320: «[...] la France a grandi et prospéré sous l'influence de la royauté, secondant le mouvement d'ascension des classes moyennes; l'Angleterre, par l'action de l'aristocratie territoriale, prenant sous sa garde les libertés du peuple.»

21. Il y aura en cela comme un effet de réciprocité : ce sera en Angleterre que l'historiographie de Guizot sera la plus appréciée. Et cela non seulement pour avoir produit une Histoire de la révolution d'Angleterre qui y restera longtemps admirée, mais d'abord pour avoir élaboré une théorie de l'histoire. C'est ce dont témoignent plusieurs articles - non signés mais généralement attribués aux grands historiens anglais - dans les revues et magazines britanniques. Ainsi, par exemple, dans son numéro de février 1844, la Revue britannique traduit et publie un article de la British and Foreign Review sur les «historiens contemporains", qui s'exprime en ces termes: "M. Guizot mérite, dans notre opinion, d'être élevé au premier rang parmi les historiens contemporains, car la philosophie de l'histoire est, selon nous, beaucoup supérieure à l'art. Sans doute M. Guizot n'a pas découvert les grandes lois qui régissent le développement de l'humanité; mais nul écrivain ne peut se vanter d'avoir plus contribué à rendre cette découverte facile. Quel autre historien a su exposer aussi bien que lui le développement des idées et des institutions humaines? en est-il un dont l'absence d'une doctrine générale propre à vérifier ses conclusions, paraisse moins gêner la marche? où trouver un penseur qui semble avoir aperçu plus clairement les causes premières de tous les phénomènes historiques, et compris avec autant de bonheur la signification particulière de chaque époque? Mieux que personne, il a lu l'histoire des idées dans l'histoire des faits.»

22. Tout comme en traductologie le fait même de traduire, l'existence de traductions confirme la possibilité de traduire malgré les croyances ou les théories contraires qui donnent la traduction pour impossible. Voir aussi le livre récent de Paul Ricœur, Sur la traduction, Paris: Bayard, 2004; en particulier les pages 26-28, 56-58, où Ricœur remet en cause l'hypothèse ethnolinguistique Whorf-Sapir.

23. Dans la suite de ce passage, Guizot met en cause la langue anglaise même: "Ce n'est point une langue systématique, régulière, rationnellement construite; elle emprunte des mots de tous côtés, aux sources les plus diverses, sans s'inquiéter de la symétrie ni de l'harmonie; elle manque de cette élégance, de cette beauté logique qui éclate dans le grec [sic], dans le latin; elle a je ne sais quelle apparence incohérente et grossière.» Ibid. p. 11.

24. Sur l'historiographie au dix-neuvième siècle - et sur les influences qui lui donnent forme - voir Paul Veyne, Comment on écrit l'histoire: essai d'épistémologie, Paris: Seuil, 1971.

25. Cet Essai a d'abord été publié en 1816 comme discours préliminaire du Musée royal (Henri Laurent, 1816-1818), et n'a été republié qu'une seule fois depuis, en 1995: La Rochelle: Rumeur des Âges.

\section{RÉFÉRENCES}

Bardoux, A. (1894) : Guizot, Paris, Hachette.

Bassnett, S. et A. Lefevere (1990): Translation, History and Culture, Londres, Pinter.

Broglie, G. de (1990): Guizot, Paris, Perrin.

Constant, B. de R. (1995): Wallstein, in Euvres complètes, Tübingen, Max Niemeyer Verlag (1809).

Gilman, M. (1925): Othello in French, Paris, Champion.

Guizot, F. (1822): De Shakspeare et de la poésie dramatique, Paris, Ladvocat.

Guizot, F. (1826): Histoire de la Révolution d'Angleterre depuis l'avènement de Charles I jusqu'à

la restauration de Charles II, Paris, A. Leroux et C. Chantpie.

Guizot, F. trad. (1828): Histoire de la chute et décadence de l'Empire romain, d'Edward GiввоN, Paris, Ledentu (1812). 
Guizot, F. (1846): Histoire générale de la civilisation en Europe depuis la chute de l'empire romain jusqu'à la Révolution française, Paris, Didier.

Guizot, F. (1847): Essais sur l'histoire de France, Paris, Charpentier (1836).

Guizot, F. (1852): Shakespeare et son temps: Étude littéraire, Paris, Didier.

Guizot, F. (1858-1867): Mémoires pour servir à l'histoire de mon temps, Paris, Michel Lévy.

Guizot, F. (1864): Histoire de la civilisation en France depuis la chute de l'Empire romain, Paris, Didier.

Guizot, F. (1887): Le temps passé: Mélanges de critique littéraire et de morale, Paris, Perrin (1809).

Guizot, F. (1995): Essai sur les limites qui séparent et les liens qui unissent les beaux-arts, La Rochelle, Rumeur des Âges (1816).

Hermans, Th. (1999): Translation in Systems: Descriptive and System-Oriented Approaches Explained, Manchester, St. Jerome.

Jones, Kathleen (1939): La Revue britannique: Son histoire et son action littéraire (1825-1840), Paris, Droz.

Larousse, Pierre éd. (1866-1879): Grand Dictionnaire Universel du XIX ${ }^{e}$ siècle, Paris, Administration du Grand Dictionnaire Universel.

Lukacs, G. (1965): Le Roman historique, Paris, Payot.

Lukacs, G. (1969): Balzac et le réalisme français, Paris, Maspero.

Moraud, M. (1933): Le Romantisme français en Angleterre de 1814 à 1848: Contribution à l'étude des relations littéraires entre la France et l'Angleterre, Paris, Honoré Champion.

Ricceur, P. (2004): Sur la traduction, Paris, Bayard.

Rosanvallon, P. (1985): Le Moment Guizot, Paris, Gallimard.

Starobinski, J. (1982): "Gibbon et la défense de l'érudition dans Le Statut de la littérature: Mélanges offerts à Paul Bénichou», Genève, Droz, p. 205-227.

Түмосzко, M.(2000): «Translation and Political Engagement: Activism, Social Change and the Role of Translation in Geopolitical Shifts, ", The Translator, vol. 6, n ${ }^{\circ}$ 1, p. 23-47.

Tymoczko, M and E. Gentzler (2002): Translation and Power, Amherst et Boston, University of Massachusetts Press.

Veyne, P. (1971): Comment on écrit l'histoire: essai d'épistémologie, Paris, Seuil.

Warsquiel, E. de, et B. Yvert (2002): Histoire de la Restauration, Paris, Perrin. 